\title{
Design of Analog Circuit for Radar Video Echo
}

\author{
Chunyu Shao, Xuezhi Zhang and Cun Xie \\ Ordnance NCO Academy, Army Engineering University, Wuhan, China
}

\begin{abstract}
Radar plays a more and more important role in modern war. Many countries have invested a lot of manpower and material resources in radar technology research and development. The radar echo is simulated with modern electronic technology and computer digital simulation technology in order to debug and test the performance of the radar system. In this paper, a simple and reliable analog circuit is designed according to the echo parameters of a certain type of radar.
\end{abstract}

Keywords-radar signal simulation; target echo and analog circuit

\section{INTRODUCTION}

In the process of developing and debugging modern radar systems, detection of radar performance and targets is an important link. If the early debugging and performance identification of the radar all use the method of external field test used in the early stage, that is to use the real target (such as aircraft) to provide the radar test signal, it not only takes a lot of manpower, financial and material resources, but also makes the development cycle lengthening. In some cases, such as bad weather, it is not even possible to achieve it. Besides, the repeatability of outfield experiments is poor and the control is more complicated. With the rapid development of electronic technology and computer digital simulation technology in recent years, the use of these technologies to simulate the radar system and environment can effectively overcome some shortcomings of the external field test flight. Therefore, radar simulation has gradually become the process of radar system performance testing with its economic, flexible and realistic characteristics. The missing method is widely used in debugging and performance evaluation of a radar subsystem.

The method of radar echo simulation can not only save a lot of manpower and material resources, but also can simulate the simulation test environment without field test, and its economy can be seen. The echo signal analog circuit is the core component of the radar echo simulation. Through the analysis above, it can be seen that the analog circuit of the radar echo signal is an effective method to assist the radar performance detection.

The radar can not radiate electromagnetic waves in the indoor teaching. It can only hit the load position, so the radar can't get the true echo of the target, which affects the teaching and can not carry out the combat operation, performance detection and troubleshooting.

\section{RESEARCH STATUS}

At present, many domestic and foreign countries have studied a variety of radar echo signal analog circuits for different applications, which can be used to accurately simulate radar echo signals in specific environments. So far, we have developed a number of analog circuits for air to ground, ground to sea, and radar signals to the sea. With the advent of highperformance radar, the requirement for radar signal simulation is increasing, which is mainly reflected in the following aspects

(1) radar simulation to digital development. (2) single channel development to multichannel. (3) it can accurately simulate the target and the surrounding environment. (4) the ability to Multiobjective output. (5) it has higher output accuracy, including target distance, speed and angle accuracy. (6) it has strong generality and can be reconfigured by software to meet the needs of different situations, such as the generation of special track, the change of signal waveform, and the change of the external environment. (7) it has good man-machine interface, easy maintenance and fault detection.

As mentioned above, the development of modern radar target echo analog circuits is a multi-functional, multi-channel and multi-target digital circuit, and it has to have the ability to accurately reflect the radar echo ability of various application conditions ${ }^{[3]}$.

This paper mainly studies the generation of signal simulation and designs the radar video echo signal generation circuit. Based on the theory of radar signal generation, the design scheme of radar video echo signal generation circuit is completed. The functional modules of the radar system are analyzed, and the functions and implementation methods of the hardware part are briefly introduced.

\section{Design Of ANALOg CiRcuit For RADAR Video Echo}

In this design circuit, the generated pulse signal has to synchronize with the radar emission trigger signal, so that the generated pulse signal can be displayed on the radar display.

In order to design high precision pulse signal generating circuit, there are usually two ways to realize it. One is to design the complex programmable logic device (CPLD) directly. The two is to use a chip of FPGA as the core, and the external RAM, $\mathrm{D} / \mathrm{A}$ converter and filter are designed and implemented.

The system finally uses the MAX II Series CPLDEPM1270T of Altera company as a digital timer, which contains 1270 logical units (LE), which supports the internal clock frequency of up to $300 \mathrm{MHz}$, in which the $\mathrm{I} / \mathrm{O}$ port is programmable, and the Schmidt trigger can be configured to improve the signal integrity, and the use of $100 \mathrm{M}$ is very convenient, and the clock source selects $100 \mathrm{M} \mathrm{Hz}$ external active crystal oscillator to reduce clock jitter and improve timing quality ${ }^{[2]}$. 
The pulse signal generation circuit is composed of single chip microcomputer, CPLD chip pulse generation module, display module, power module and so on. As shown in FIGURE1.

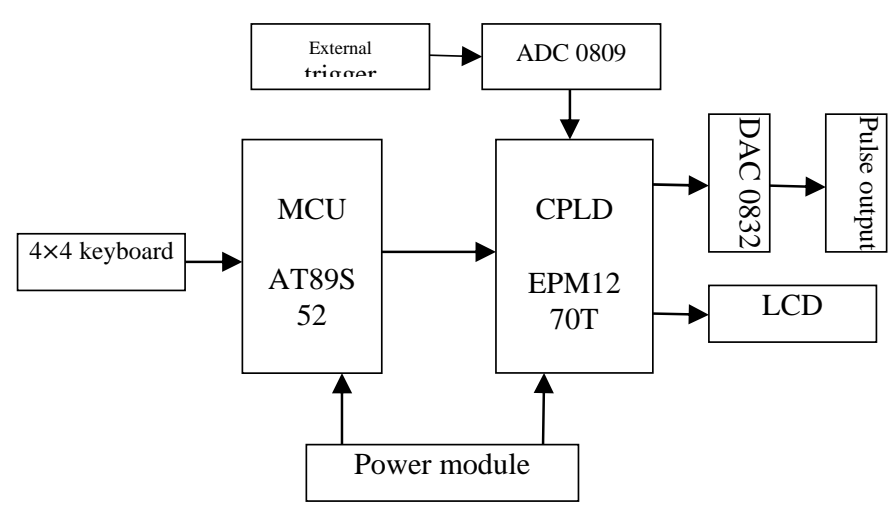

FIGURE I. HARDWARE CIRCUIT DIAGRAM OF SIGNAL GENERATING CIRCUIT

The single chip microcomputer is used to control and process the whole system. The frequency of the signal and the initial phase value are input by the keyboard (4X4) connected with the single chip microcomputer. After the microprocessor processing, the signal is converted to the CPLD chip through the interface circuit, and then the required pulse signal can be obtained by the CPLD pulse generator. The frequency value of the signal is calculated and displayed through LCDI602.

There are two triggering modes in the CPLD pulse generator: one is the internal trigger mode, the trigger signal is generated within the CPLD; the other is an external trigger mode, and the trigger signal comes from the external synchronization device.

In this design circuit, the trigger signal is selective, not all the signals can be used, so we can use the launch trigger pulse generated by the radar itself (the pulse width of $4.8 \mathrm{~s}$, the amplitude not less than $12 \mathrm{~V}$ ) as the external trigger signal of the CPLD pulse generator, so that the pulse signals can be produced. The pulse signal can be displayed on the radar display synchronously with the trigger signal of radar transmitting.

\section{A. Control Module}

AT89S52 is a low-power, high-performance CMOS 8 bit microcontroller with $8 \mathrm{~K}$ system programmable Flash memory. It has 32 programmable I/O lines, fully compatible with the instructions and pins of the industrial 51 MCU, full duplex serial channel, interruption and wake-up functions after power failure.

\section{1). Design block figure \\ 2). Circuit analysis}

The system uses AT89S52 as the control core. The 8 pins of the P1 port are connected to the $4 \times 4$ matrix keyboard by the line line. The function of step mode selection, sleep and wakeup, step and subtract, etc. is completed according to the key situation. The P0.0 to P0.7 ports are connected to the I/O port of CPLD with 8 core lines. P2.7.P3.6.OE2 and P3.7 even OE1, 30 foot are controlled, thus the output of the corresponding frequency pulse signal is realized. As shown in FIGURE 2.

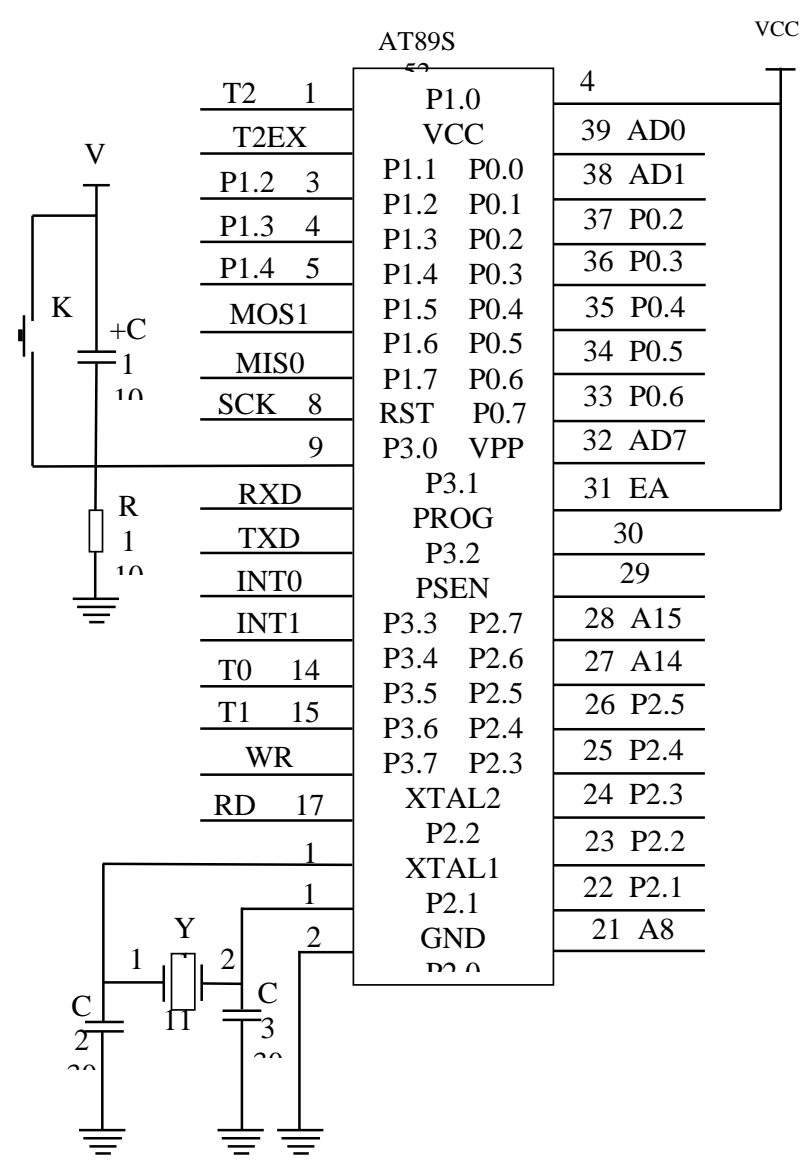

FIGURE II. CONTROL CIRCUIT DIAGRAM

\section{B. Display Module}

1). Design block figure

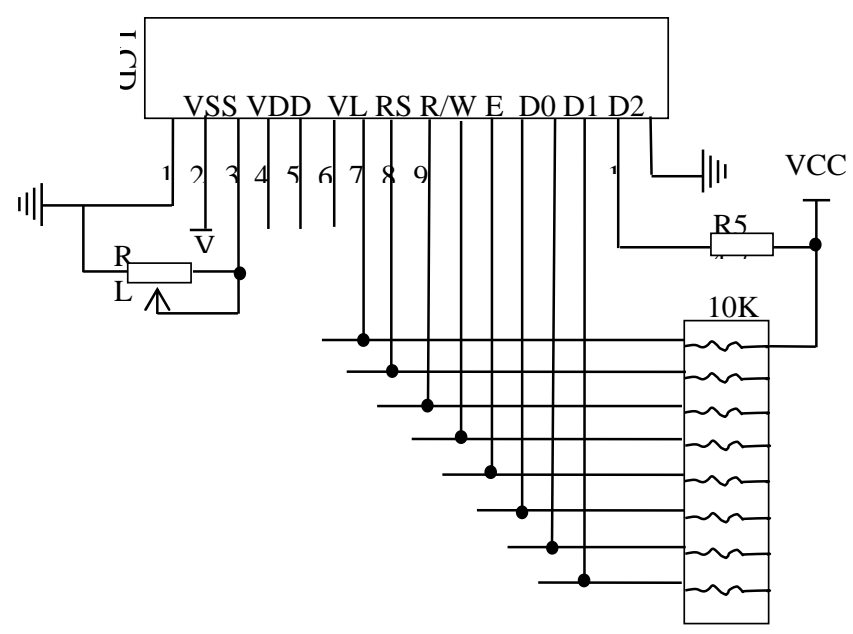

FIGURE III. LCD1602 CONNECTION GRAPH 
LCD1602 is a character LCD. It is a $16^{*} 2$ display (32 characters) and each character is composed of $5 * 7$ dot matrix.

\section{2). Circuit analysis}

\section{a) 1602 use of introduction}

In the monolithic integrated circuit system, in order to achieve the good human-machine interface, besides needing the keyboard and so on input equipment, generally also has the display output equipment. Commonly used monitors are: LED display, LED for short, liquid crystal display (LCD). LED display and LCD display have the characteristics of simple structure, low cost, flexible configuration and convenient interface with MCU. This system is displayed by 1602LCD.

\section{b) 1602 hardware connection introduction}

The 7 to 14 pins of the 1602 are connected to the general $\mathrm{I} / \mathrm{O}$ port of CPLD, and the system hardware connection is shown in FIGURE $3^{[1]}$.

\section{Keyboard Input Module}

The matrix keyboard is also called the row and column keyboard. It is a keyboard composed of $4 \mathrm{I} / \mathrm{O}$ lines as lines and $4 \mathrm{I} / \mathrm{O}$ lines as columns. At each intersection point of the line and the column, a key is set up. In this way, the number of keys in the keyboard is $4 \times 4$. This determinant keyboard structure can effectively improve the utilization rate of $\mathrm{I} / \mathrm{O}$ port in the SCM system.

\section{1). Design block figure}

First, the high four bit output from the P1 port outputs low level, low four bit output high level, and reads the keyboard state from the low four bit of the P1 port. From the low four bit output of the P1 port to the low level, the high four bit output level is high, and the keyboard state is read from the high four bit of the P1 port. By combining the two reading results, we can get the feature encoding of the current key.

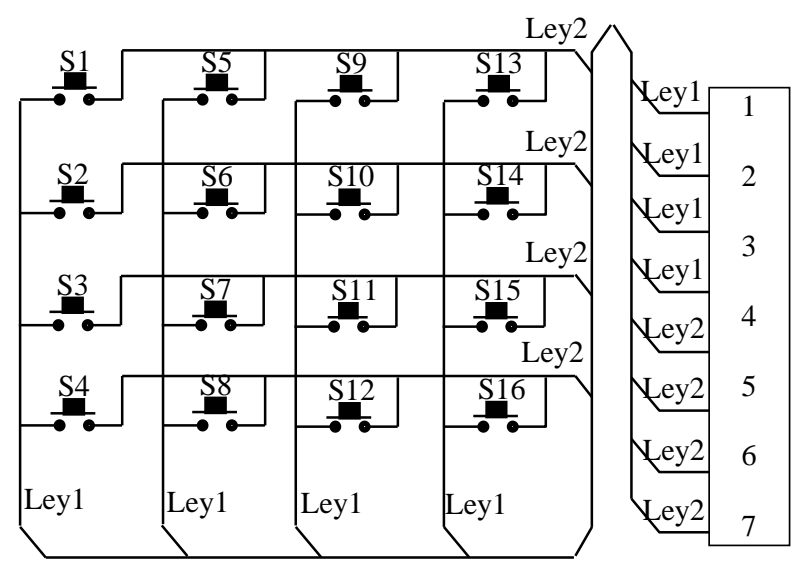

FIGURE IV. KEYBOARD CONNECTION DIAGRAM

\section{2). Circuit analysis}

a) Keyboard hardware connection

The P1.O to P1.7 ports in the single chip system area are connected to the Ley1O to Leyl3, Ley20 to Ley23 ports in the
" $4 \mathrm{x} 4$ determinant keyboard" area with 8 core line lines; the wiring diagram is shown in FIGURE 4.

\section{b) 4*4 matrix keyboard recognition processing}

By defining the row values and column values of each key and combining them, the key codes of each key are obtained. The line value and column value of the keyboard matrix are sent to the parallel port of the single chip microcomputer. One end of the key is sent to VCC by a current limit resistance, and the single chip sends a low level to the earth through the program. Through the keyboard processing program, to determine whether there is a press button, and which key is pressed, what is its corresponding function? Because the key is jitter in the instant of pressing the key, in order to prevent misoperation, the program must be processed to shake. One of the two parallel ports, one of which output scanning code, makes the key step by line to earth, another parallel port reads the key information. The key is identified by the line scan value and the feedback signal, and the function of the key is detected by the software lookup.

\section{Power Module}

\section{1). Design block figure}

In this system, a power supply is used: AT89S52 and pulse signal generation module are supplied by $+5 \mathrm{~V}$. The voltage output circuit uses a step-down switch type integrated voltage regulator LM2576, which can reduce the input voltage of the voltage regulated power supply by using the series filter inductor, which can reduce the input voltage of the voltage regulated power supply. The hardware circuit is shown in FIGURE 5.

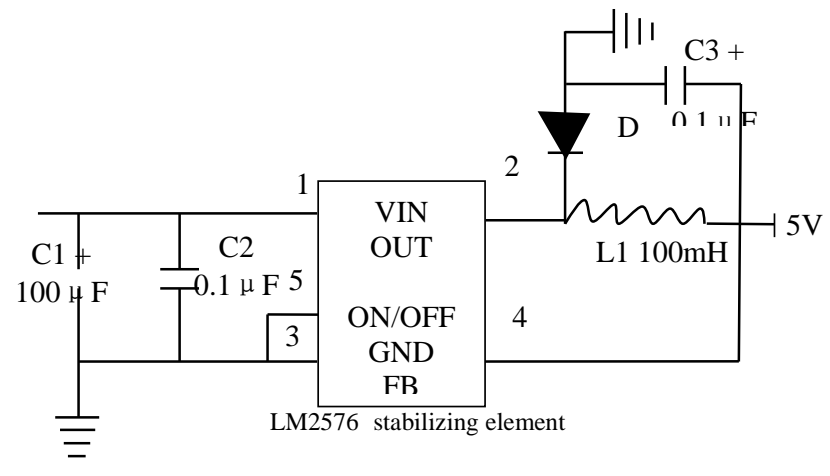

FIGURE V. POWER CIRCUIT DIAGRAM

\section{2). Circuit analysis}

The LM2576 includes $52 \mathrm{~Hz}$ oscillator, $1.23 \mathrm{~V}$ reference voltage regulator, thermal correlation circuit, current limiting circuit, amplifier, comparator and internal voltage stabilizing circuit. In order to produce different output voltages, the negative end voltage $(1.23 \mathrm{~V})$ of the comparator is usually connected to the voltage resistance network at the positive end, so that different values can be selected according to the difference of the output voltage, and the resistance has been adjusted accurately in the chip based on the different models. The output voltage divider resistance network is compared with the internal reference voltage. If the voltage is biased, the 
amplifier can control the output duty ratio of the internal oscillator, thus keeping the output voltage stable.

The characteristic of this circuit is simple structure. The components used are quite common, and it is easy to repair when troubles happen.

\section{E. Pulse Generator Module}

This module is designed to generate the pulse signal needed for radar. It should synchronize with the radar signal, and the duty ratio of the video echo signal is $0.1 \%$. The general circuit is difficult to produce. Therefore, the CPLD pulse generator is used to produce the required signal.

The minimum pulse width depends on the frequency of the main clock. The range of duty ratio depends on the frequency of the synchronous pulse. The higher the frequency of the clock signal, the narrower the minimum pulse width of the output pulse is, the higher the resolution of the pulse. When the synchronous pulse signal takes different frequency values, the range of the duty ratio and the precision of the output pulse are different. The lower the frequency of the synchronous pulse signal, the greater the range of the duty ratio of the output pulse and the higher the precision.

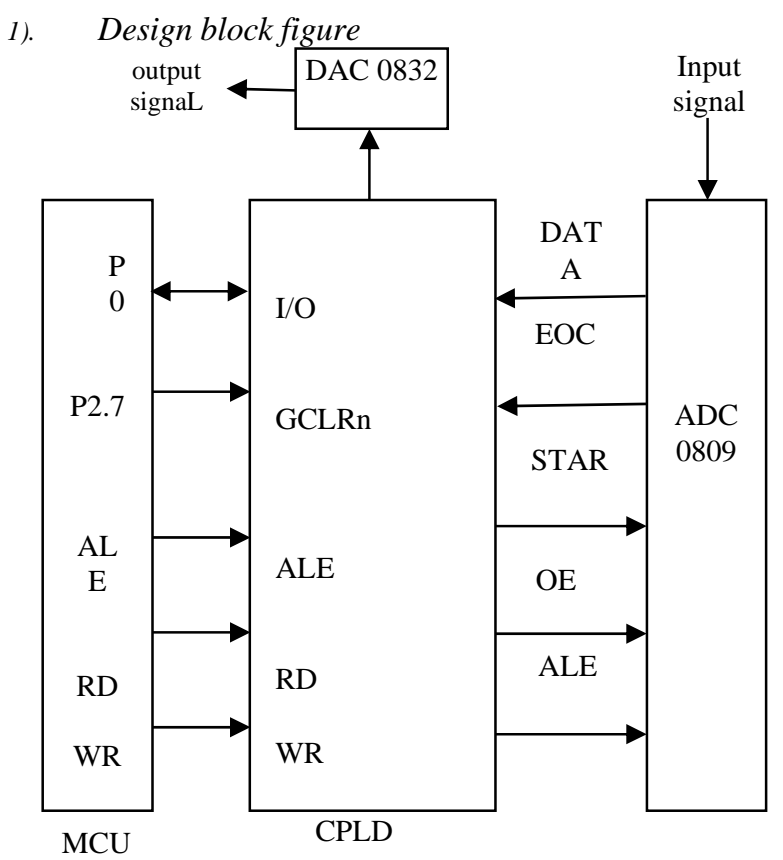

FIGURE VI. CPLD CONNECTION DIAGRAM

The I/O foot of CPLD can be allocated at will. We can adjust pin distribution according to need.

EPM1270T data bus P0 port is connected with CPLD general I/O port to complete data and low 8 bit address transmission. The control bus includes MCU read-write signal RD (P3.7) and WR (P3.6) and address latch signal ALE and high address line A15 (P2.7), through the global signal input of the CPLD, including global clockwise input, global Zero input INPOT/GCLRn, global input INPUT/OE1, INPUT/OE2. These signals are connected to each macrocell in CPLD by dedicated cable, ensuring that the signal is equal to the delay of each macrocell and is the shortest.

\section{2). Circuit analysis}

The I/O foot of CPLD can be allocated at will. We can adjust pin distribution according to need.

EPM1270T data bus P0 port is connected with CPLD general I/O port to complete data and low 8 bit address transmission. The control bus includes MCU read-write signal RD (P3.7) and WR (P3.6) and address latch signal ALE and high address line A15 (P2.7), through the global signal input of the CPLD, including global clockwise input, global Zero input INPOT/GCLRn, global input INPUT/OE1, INPUT/OE2. These signals are connected to each macrocell in CPLD by dedicated cable, ensuring that the signal is equal to the delay of each macrocell and is the shortest.

\section{SUMMARY}

The video echo analog circuit designed in this paper consists of five parts: the control module, the display module, the keyboard input module, the power module and the pulse generator module. The pulse generator module is the core of the circuit, which mainly produces the required pulse signal. As shown in FIGURE 7, the pulse signal waveform generated by the analog circuit of the video echo is designed.

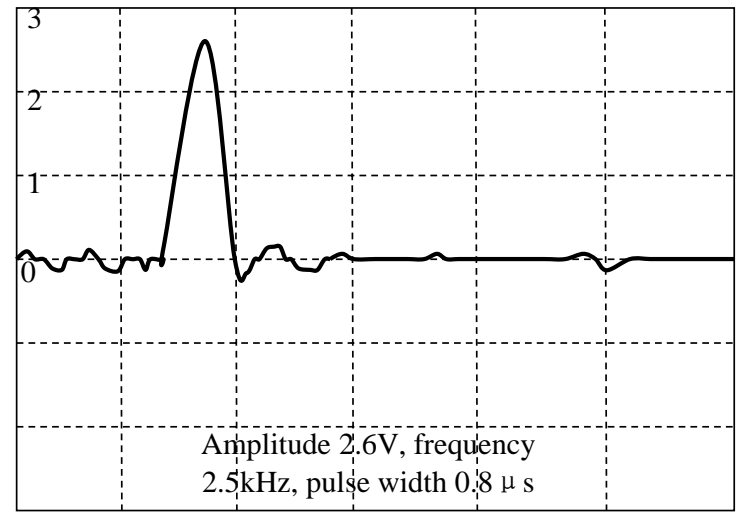

\section{FIGURE VII. THE WAVEFORM DIAGRAM OF THE GENERATED} PULSE SIGNAL

The design of this circuit not only solves the difficult problem of obtaining the target echo in the radar room, but also provides a certain basis for the troubleshooting of the video echo line. It helps to improve the operator's combat operation and troubleshooting ability, thus promoting radar course teaching.

\section{REFERENCE}

[1] Tian Kai; 51 MCU +CPLD makes the system more efficient; electronic technology, November 2011.

[2] Changsheng; multichannel digital delay pulse generator based on CPLD and DS1020; "electronic technology application" 01 issue in 2015. 
[3] Xu Wenqiang; research on Key Technologies of radar video echo simulation; dissertation of North Central University; 2007. 